\title{
Olfactory functions are not associated with autism severity in autism spectrum disorders
}

\author{
This article was published in the following Dove Press journal: \\ Neuropsychiatric Disease and Treatment \\ 26 November 2013 \\ Number of times this article has been viewed
}

\section{Iva Dudova \\ Michal Hrdlicka}

Department of Child Psychiatry, University Hospital Motol, Prague, Czech Republic
Correspondence: Iva Dudova Department of Child Psychiatry, Charles University Second Faculty of Medicine, University Hospital Motol, 84 V Uvalu, Prague 15006, Czech Republic

Tel +420 224433458

Fax +420 224433420

Email iva.dudova@lfmotol.cuni.cz
Background: Changes in olfactory functions have been found in many neurodegenerative and psychiatric disorders, including autism spectrum disorders (ASDs). The aim of the present study was to evaluate the relationship between olfactory functions (odor-detection thresholds, odor identification, and odor preference) and autism severity and sensory-related behavior in children and adolescents with ASD.

Subjects and methods: Our sample consisted of 35 high-functioning patients with ASD (mean age 10.8 \pm 3.6 years, 31 boys). Olfactory testing (threshold and identification) used the Sniffin' Sticks test. Odor pleasantness was assessed on a 5-point scale using the Identification part of the Sniffin' Sticks test. The severity of autistic psychopathology was measured using the Childhood Autism Rating Scale (CARS).

Results: Using Spearman's correlation, we found no significant correlations between autism severity (as expressed by total CARS score) and odor-detection thresholds ( $R=0.144, P=0.409$ ), odor identification $(R=0.07, P=0.966)$, or odor pleasantness $(R=-0.046, P=0.794)$. There was also no significant relationship between CARS item 9 ("Taste, smell, and touch response and use") and odor-detection thresholds $(R=0.170, P=0.330)$, odor identification $(R=0.282, P=0.100)$, or odor pleasantness $(R=0.017, P=0.923)$.

Conclusion: We did not find any significant relationship between the severity of autistic psychopathology and olfactory functions.

Keywords: autism spectrum disorders, psychopathology, Sniffin' Sticks, odor threshold, odor identification, odor pleasantness

\section{Introduction}

Changes in olfactory functions have been found in many neurodegenerative and psychiatric disorders, including Parkinson's disease, Alzheimer-type dementia, schizophrenia, obsessive-compulsive disorder, and attention deficit/hyperactivity disorder (ADHD), as well as autism spectrum disorders (ASDs). ${ }^{1,2}$ In a recent systematic review of olfaction in child and adolescent psychiatric disorders, Schecklmann et $\mathrm{al}^{2}$ emphasized that specific changes in olfactory function were found mainly in disorders with a dopaminergic pathology (eg, ADHD, autism, schizophrenia, and 22q11-deletion syndrome). Unequivocal findings in early onset schizophrenia showed diminished discrimination and identification with unaltered sensitivity, and thus olfactory identification was seen as a highly promising biomarker candidate for schizophrenia. Patients with 22q11-deletion syndrome featured severe olfactory impairments in all olfactory domains; however, when the authors statistically controlled the results for craniofacial abnormalities, which might contribute to diminished olfactory findings, 
no significant olfactory abnormalities were found. Studies investigating ADHD and autism relative to olfaction have produced inconsistent results. ${ }^{2}$

There have been few studies to deal with olfactory abnormalities in ASD. Consistent results came from adult and predominantly adolescent studies. Suzuki et $\mathrm{al}^{3}$ measured odordetection threshold and odor identification in twelve adult males with Asperger's syndrome (AS) and twelve matched control subjects using the Cain two-bottle test and the University of Pennsylvania Smell Identification Test (UPSIT). AS subjects did not have impaired odor detection, but did have significantly impaired olfactory identification. Bennetto et $\mathrm{al}^{4}$ focused on olfactory identification and compared 21 participants with autism (age 10-18 years) with 27 well-matched controls. Using the Sniffin' Sticks test, they found that olfactory identification was significantly impaired among participants with autism. Tavassoli and Baron-Cohen ${ }^{5}$ examined 38 adult participants with ASD and 42 controls, and found no difference in olfactory detection thresholds between the groups.

Pediatric studies yielded different results. Brewer et $\mathrm{al}^{6}$ investigated olfactory identification in 15 children with high-functioning autism (HFA; mean age 77.8 months) and 15 controls (matched for age, sex, and intelligence quotient) utilizing a modified visual analog of the UPSIT. No significant differences were found between the two groups regarding olfactory identification ability. However, smell identification was negatively correlated with age in the HFA group, a finding that was opposite to the relationship found in controls. May et $\mathrm{al}^{7}$ reported results of a 5-year follow-up of children with HFA that followed a previous study. They found that both HFA and controls had improved birhinal olfactory identification. Additionally, unirhinal olfactory identification was compared between HFA, AS, and controls (twelve participants per group). Unirhinal olfactory identification was reduced in HFA compared to AS and controls, suggesting an orbitofrontal compromise in HFA, but orbitofrontal integrity in AS.

In our previous reports, we found children and adolescents with ASD, in comparison to healthy controls, being significantly impaired relative to odor-detection thresholds (6.3 vs $7.9, P=0.025$ ). There was no significant difference between autistic and control subjects on the total score of olfactory identification (10.7 vs $10.6, P=0.799) .{ }^{8}$ Autistic subjects assessed all odors as overall less pleasant than control subjects' assessment; however, the difference was not significant (45.1 vs 42.0, $P=0.123$ ). ${ }^{9}$

The aim of the present study was to analyze further the data of the autistic group from our previous research in order to determine if and to what extent a relationship between olfactory functions (odor-detection thresholds, odor identification, and odor preference) and autism severity and sensoryrelated psychopathology exists in children with ASD.

\section{Subjects and methods Participants}

Subjects with ASD were recruited from the Department of Child Psychiatry, University Hospital Motol (Prague). The study was approved by the Ethics Committee of the University Hospital Motol. All participants and their legal guardians agreed to participate in the study; legal guardians signed informed consents for subjects under 18 years of age, as per Czech law. Only one subject, aged 18.5 years, personally signed the consent.

Inclusion criteria for autistic subjects were: 1) diagnosis of AS, high-functioning autism, or other high-functioning ASD, confirmed using the Autism Diagnostic Interview Revised (ADI-R) ${ }^{10}$ and/or the Childhood Autism Rating Scale (CARS); ${ }^{11}$ 2) age 6-18 years; and 3) an intelligence quotient $\geq 70$. Subjects with organic smell disturbances and/or acute respiratory infection were excluded.

Our sample consisted of 35 autistic patients with a mean age of 10.8 \pm 3.6 years (range 6.1-18.5 years, 31 boys). Diagnoses made according to the International Classification of Diseases (10th revision) ${ }^{12}$ included 27 patients with AS, five patients with childhood autism, and three patients with unspecified pervasive developmental disorders. All participants were nonsmokers.

\section{Materials and procedure}

Olfactory threshold and identification were examined using Sniffin' Sticks, a commercially available three-part olfaction test (Burghardt Medical Technology, Wedel, Germany): Threshold, Discrimination, and Identification. The test has been reliably standardized, is appropriate for both adults and children, ${ }^{13,14}$ and has been previously used in the Czech population. ${ }^{15}$ Two parts of the test (Threshold and Identification) were used in this study. We also developed a simple method to assess odor pleasantness. ${ }^{9}$ Odor pleasantness was assessed using the Identification part of the Sniffin' Sticks test on a 5-point scale, with 1 being most pleasant, 3 being neutral, and 5 being most unpleasant. We supplemented the scale with emoticons, which was thought to be useful in autistic children, in order to facilitate decisions.

During testing, odorants were presented using modified felt-tip pens. For odor presentation, the cap was removed by the investigator and the pen tip was placed centrally, 
approximately $2 \mathrm{~cm}$ in front of the nose. The tests were administered in sequence starting with testing of odor thresholds, followed by testing of odor identification (this sequence matched the manufacturer's guidelines). Finally, odor pleasantness was examined. For more details on testing procedures, please see the detailed description in Dudova et al. ${ }^{8}$

The severity of autistic psychopathology was measured using the CARS. ${ }^{11}$ For calculations, we used the total score and the score of item 9 ("Taste, smell, and touch response and use"), which was directly connected to olfactory behavioral abnormalities.

\section{Data analysis}

Statistical analysis was performed using SPSS version 21.0 (IBM, Armonk, NY, USA). Descriptive statistics for the sample were used. The Spearman correlation analysis was used to explore relationships between autistic psychopathology and odor-detection threshold, odor identification total score, and odor pleasantness total score.

\section{Results}

The mean CARS score in our sample was $36.3 \pm 3.6$ (range 30.0-43.5). The mean score on CARS item 9 was $2.2 \pm 0.6$ (range 1.0-3.5). As we published previously, the mean odordetection threshold was found to be $6.3 \pm 3.1$, mean total score of olfactory identification was $10.7 \pm 2.6,{ }^{8}$ and mean total score of odor pleasantness was $45.1 \pm 8.2$. $^{9}$

We found no significant correlations between autism severity (as expressed by the total CARS score) and odordetection thresholds, odor identification, or odor pleasantness. There was no significant relationship either between the CARS item 9 score ("Taste, smell, and touch response and use") and odor-detection thresholds, odor identification, or odor pleasantness. For details, see Table 1.

\section{Discussion}

Chronologic aging plays an important role in olfactory research in autism. As we demonstrated in the introductory part of our article, pediatric results ${ }^{6-8}$ differed from results

Table I Correlations of olfactory functions and autistic psychopathology in children and adolescents with autism spectrum disorder

\begin{tabular}{lll}
\hline & Total CARS score & CARS item 9 \\
\hline Odor-detection threshold & $R=0.144, P=0.409$ & $R=0.170, P=0.330$ \\
Odor identification & $R=0.007, P=0.966$ & $R=0.282, P=0.100$ \\
Odor pleasantness & $R=-0.046, P=0.794$ & $R=0.017, P=0.923$ \\
\hline Abbreviation: CARS, Childhood Autism Rating Scale. &
\end{tabular}

obtained from studies involving adolescents ${ }^{4}$ and adults. ${ }^{3,5}$ Brewer et $\mathrm{al}^{6}$ suggested that the absence of an olfactory identification deficit in HFA was caused by immature olfactory identification at younger ages. They formulated the hypothesis that those with HFA may appear to "grow into the deficit" as they mature and enter puberty; in other words, olfactory identification abilities in HFA children will appear to deteriorate with age, but only when compared to controls. This was confirmed, in some HFA participants, in a 5-year follow-up of children with HFA by May et al. ${ }^{7}$ They found that olfactory identification improved in both groups (HFA and controls) during the follow-up, but development was more heterogeneous in children with HFA. The term heterogeneous is at best rather vague, and our interpretation of it is that most if not all controls had improved odor identification over time, while those with HFA had mixed results, ie, with some showing improvement with age, and others not. Based on this interpretation of the data, May et al's results agree with our previous results, which were obtained from a group of participants of comparable age. We found odor-identification ability to be positively correlated with age in the control group, but not in the autism group. ${ }^{8}$ It is reasonable to conclude that chronologic aging contributes to some of the problems encountered during olfactory research associated with autism.

To our knowledge, there has been only one study that explored the relationship between autistic psychopathology and olfactory functions. However, the methodology was different, and thus the results are not directly comparable. Bennetto et $\mathrm{al}^{4}$ examined olfactory and taste functioning in individuals with autism to determine if there was a connection between olfaction and social impairment in autism. They examined the relationship between olfactory identification and comparable scores taken from the ADI-R, the standardized parent interview for autism. In participants with autism, olfactory identification was marginally related to their ability to engage in social verbalization or chatting, and significantly related to their skill at maintaining a reciprocal conversation. Children with a poor performance on the olfactory identification test were more likely to have greater social impairment.

Generally, it can be said that studies attempting to correlate autism symptoms and/or severity with various neurophysiological abnormalities have not been entirely successful. ${ }^{16}$ This is not only with regard to olfactory functions. For example, inconsistent results have also been obtained in research looking at electroencephalogram (EEG) abnormalities and skin-conductance reactions. Some studies found a positive 
association between EEG abnormalities and autism severity, ${ }^{17}$ while some did not. ${ }^{18,19}$ Similarly, some studies found abnormal skin-conductance reactions in autism, ${ }^{20,21}$ while others found no relationship. ${ }^{22}$ The probable interpretation of our negative results is that olfactory abnormalities in autism represent a trait marker for the disorder rather than a state marker.

Our results are limited by sample size and the age diversity of the study group, which included children as well as adolescents. Most of our patients were diagnosed with AS (27 of 35 patients), which is atypical and does not represent the most common diagnosis in the ASD group. ${ }^{23}$ Another limitation was the use of the CARS, which is a quite simple rating scale, and the behavior related to sensory input was concentrated into just one item. However, similar methodology using the CARS has already been successfully used in other autism studies. ${ }^{18,24}$ In future, the possible relationship between sensory-related behavior and olfactory functions could be better examined by using more sophisticated scales, such as the Infant/Toddler Sensory Profile. ${ }^{25}$

\section{Conclusion}

We did not find any significant relationship between the severity of autistic psychopathology and odor-detection thresholds, odor identification, or odor pleasantness.

\section{Acknowledgments}

This study was supported by the Ministry of Education, Youth and Sports, Czech Republic (research grant COST LD11028) and by the Ministry of Health, Czech Republic (conceptual development of research organization, University Hospital Motol, Prague, Czech Republic 00064203). The authors would like to thank Thomas Secrest, MSc for his assistance with the English version of the manuscript.

\section{Disclosure}

The authors report no conflicts of interest in this work.

\section{References}

1. Brewer WJ, Castle D, Pantelis C. Olfaction and the Brain. 1st ed. New York: Cambridge University Press; 2006.

2. Schecklmann M, Schwenck C, Taurines R, et al. A systematic review on olfaction in child and adolescent psychiatric disorders. J Neural Transm. 2013;120:121-130.

3. Suzuki Y, Critchley HD, Rowe A, Howlin P, McMurphy DG. Impaired olfactory identification in Asperger's syndrome. J Neuropsychiatry Clin Neurosci. 2003;15:105-107.

4. Bennetto L, Kuschner ES, Hyman S. Olfaction and taste processing in autism. Biol Psychiatry. 2007;62:1015-1021.

5. Tavassoli T, Baron-Cohen S. Olfactory detection thresholds and adaptation in adults with autism spectrum condition. J Autism Dev Disord. 2012;42:905-909.
6. Brewer WJ, Brereton A, Tonge BJ. Dissociation of age and ability on a visual analogue of the University of Pennsylvania Smell Identification Test in children with autism. Res Autism Spectr Disord. 2008;2: 612-620.

7. May T, Brewer WJ, Rinehart NJ, Enticott PG, Brereton AV, Tonge BJ. Differential olfactory identification in children with autism and Asperger's disorder: a comparative and longitudinal study. J Autism Dev Disord. 2011;41:837-847.

8. Dudova I, Vodicka J, Havlovicova M, Sedlacek Z, Urbanek T, Hrdlicka M. Odor detection threshold, but not odor identification, is impaired in children with autism. Eur Child Adolesc Psychiatry. 2011;20: 333-340.

9. Hrdlicka M, Vodicka J, Havlovicova M, Urbanek T, Blatny M, Dudova I. Brief report: Significant differences in perceived odor pleasantness found in children with ASD. J Autism Dev Disord. 2011;41:524-527.

10. Lord C, Rutter M, LeCouteur A. Autism diagnostic interview - revised: a revised version of a diagnostic interview for caregivers of individuals with possible pervasive developmental disorders. JAutism Dev Disord. 1994;24:659-685.

11. Schopler E, Reichler RJ, DeVellis RF, Daly K. Toward objective classification of childhood autism: childhood autism rating scale (CARS). J Autism Dev Disord. 1980;10:91-103.

12. World Health Organization. International Classification of Diseases. 10th ed. Geneva: WHO; 1992.

13. Hummel T, Sekinger B, Wolf SR, Pauli E, Kobal G. 'Sniffin' sticks': olfactory performance assessed by the combined testing of odor identification, odor discrimination and olfactory threshold. Chem Senses. 1997;22:39-52.

14. Kobal G, Klimek L, Wolfensberger M, et al. Multicenter investigation of 1,036 subjects using a standardized method for the assessment of olfactory function combining tests of odor identification, odor discrimination, and olfactory thresholds. Eur Arch Otorhinolaryngol. 2000;257:205-211.

15. Vodicka J, Pellant A, Chrobok V. Screening of olfactory function using odourized markers. Rhinology. 2007;45:164-168.

16. Kulisek R, Hrncir Z, Hrdlicka M, et al. Nonlinear analysis of the sleep EEG in children with pervasive developmental disorder. Neuroendocrinol Lett. 2008;29:512-517.

17. Ekinci O, Arman AR, Isik U, BezY, Berkem M. EEG abnormalities and epilepsy in autistic spectrum disorders: clinical and familial correlates. Epilepsy Behav. 2010;17:178-182.

18. Hrdlicka M, Komarek V, Faladova L, et al. EEG abnormalities are not associated with symptom severity in childhood autism. Stud Psychol (Bratisl). 2004;46:229-234.

19. Hartley-McAndrew M, Weinstock A. Autism spectrum disorder: correlation between aberrant behaviors, EEG abnormalities and seizures. Neurology Int. 2010;2:e10.

20. Kylliainen A, Hietanen JK. Skin conductance responses to another person's gaze in children with autism. J Autism Dev Disord. 2006;36: $517-525$.

21. Joseph RM, Ehrman K, McNally R, Keehn B. Affective response to eye contact and face recognition ability in children with ASD. $J$ Int Neuropsychol Soc. 2008;14:947-955.

22. Ben Shalom D, Mostofsky SH, Hazlett RL, et al. Normal physiological emotions but differences in expression of conscious feelings in children with high-functioning autism. J Autism Dev Disord. 2006;36: 395-400.

23. Chiappedi M, Rossi G, Rossi M, Bejor M, Balottin U. Autism and classification systems: a study of 84 children. Ital $J$ Pediatr. 2010;36:10

24. Guerini FR, Bolognesi E, Chiappedi M, et al. SNAP-25 single nucleotide polymorphisms are associated with hyperactivity in autism spectrum disorders. Pharmacol Res. 2011;64:283-288.

25. Dunn W. Infant/Toddler Sensory Profile User's Manual. San Antonio: Psychological Corporation; 2002. 
Neuropsychiatric Disease and Treatment

Dovepress

\section{Publish your work in this journal}

Neuropsychiatric Disease and Treatment is an international, peerreviewed journal of clinical therapeutics and pharmacology focusing on concise rapid reporting of clinical or pre-clinical studies on a range of neuropsychiatric and neurological disorders. This journa is indexed on PubMed Central, the 'PsycINFO' database and CAS

The manuscript management system is completely online and includes a very quick and fair peer-review system, which is all easy to use. Visit http://www.dovepress.com/testimonials.php to read real quotes from published authors.

Submit your manuscript here: http://www.dovepress.com/neuropsychiatric-disease-and-treatment-journal 